\title{
CONVERGENCIA ODS-UNIVERSIDAD. UNA PROPUESTA PARA CONOCER LAS EXPECTATIVAS Y PERCEPCIONES DE LA COMUNIDAD ACADÉMICA ACERCA DE LA AGENDA 2030
}

\author{
Cavallo, Marcela Analía \\ Ledesma, Alicia Beatriz \\ Díaz, Liliana Patrícia \\ Facco; Silvina María del Luján \\ Benzi, Carolina Soledad \\ Schmidt Strano, Erika
}

\section{Resumen:}

En este escrito se presenta el proyecto de investigación denominado: "Aportes de la Universidad a la Agenda 2030. El impacto de las percepciones y expectativas de la comunidad académica de la Facultad de Ciencias Económicas y Estadística (UNR) en la consecución de los Objetivos de Desarrollo Sostenible (ODS)" que será desarrollado en el período 2020-2024. El problema que guía nuestro trabajo, tiene como punto de partida a la Universidad, en su rol formativo y generador de conocimiento, pero principalmente como agente clave en el desarrollo social para responder a las demandas en pos de un mundo más equitativo y sostenible. Asimismo, un valioso aporte han sido algunos trabajos como los conceptos de la Red de Australia, Nueva Zelanda y el Pacífico de soluciones de desarrollo sostenible (SDSN) en pos de la promoción de soluciones políticas y de educación pública para la sostenibilidad y el recorrido por algunas investigaciones que dan cuenta de un mapa preliminar sobre la presencia de los ODS en universidades argentinas. Con este marco, el escrito presenta la propuesta de investigación sobre el tema en la Facultad de Ciencias Económicas y Estadística para los 4 años de duración del proyecto. Se sostiene que los resultados de la investigación permitirán contribuir al enriquecimiento de los debates en torno de los ODS. Asimismo, los hallazgos producidos sobre percepciones y expectativas, podrán ser herramientas de gestión en la unidad académica de referencia en cuanto permitirán problematizar la construcción de modelos, el diseño de políticas y estrategias en pos del logro de los ODS.

Palabras clave: Agenda 2030- Objetivos de Desarrollo Sostenible- Universidad 


\section{Abstract}

This paper introduces the research project called: "University Contributions to the 2030 Agenda. The impact of the perceptions and expectations of the academic community of the Faculty de Ciencias Económicas y Estadística (UNR) in the achievement of the Development Goals Sustainable Development (ODS)" that will be developed in the period 2020-2024. The problem that guides our work has its starting point at the University in its formative and knowledge-generating role, but mainly as a key agent in social development to respond to the demands for a more equitable and sustainable world. Likewise, some works, such as the concepts of the Australia, New Zealand and Pacific Network of Sustainable Development Solutions (SDSN) in pursuit of the promotion of political solutions and public education for sustainability and the route through some investigations that reveal a preliminary map on the presence of the SDGs in argentine universities, have been a valuable contribution. Within this framework, this paper presents the 4-years' research proposal at the Facultad de Ciencias Económicas y Estadística hoping the results will contribute to enriching the debates around the SDGs. At the same time, the findings produced on perceptions and expectations may be considered as management tools in the academic unit of reference as they will allow to problematize the construction of models, the design of policies and strategies in pursuit of the achievement of the SDGs.

Keywords: Agenda 2030- Sustainable Development Goals- University

\section{INTRODUCCIÓN}

En este escrito se presenta la propuesta del proyecto de investigación denominado: Aportes de la Universidad a la Agenda 2030. El impacto de las percepciones y expectativas de la comunidad académica de la Facultad de Ciencias Económicas y Estadística (UNR) en la consecución de los Objetivos de Desarrollo Sostenible1.

El problema que guía nuestro trabajo, tiene como punto de partida a la Universidad, en su rol formativo y generador de conocimiento pero principalmente como agente clave en el desarrollo social para responder a las demandas en pos de un mundo más equitativo y sostenible. Asimismo, algunos antecedentes han sido una importante guía en este trabajo. Por un lado, los conceptos de la Red de Australia, Nueva Zelanda y el Pacífico de soluciones de desarrollo sostenible (SDSN) en pos de la promoción de soluciones

1 Proyecto de investigación período 2020-2023 integrado por: Cavallo, Marcela; Ledesma, Alicia; Diaz, Liliana; Facco, Silvina; Schmidt Strano, Erika y Benzi, Carolina. 
políticas y de educación pública para la sostenibilidad. Por el otro, el recorrido por algunas investigaciones exploratorias que dan cuenta de un mapa preliminar sobre la presencia de los ODS en universidades argentinas.

En este marco cobra relevancia el rol fundamental que compete a la Universidad en la incorporación de la educación para la sostenibilidad. En esta dirección, si bien este enfoque comenzó hace casi tres décadas, fue recién en la Conferencia Mundial de la UNESCO del 2009 donde se validaron los intereses de universitarios en introducir la sostenibilidad a cuestiones ligadas a la investigación, la gestión y las relaciones con la sociedad. Así como existen numerosos y válidos argumentos en favor de la responsabilidad social que la empresa debe asumir para minimizar o reparar los daños sociales y ambientales perpetrados durante décadas, la efectiva incorporación de la noción de responsabilidad social organizacional (RSO), y por ende de la sostenibilidad, a las currículas de grado parece no haber logrado sustanciarse.

A partir de estos argumentos es que en la actualidad se impone en todas las carreras universitarias, y particularmente en las del campo de las ciencias económicas, repensar las consecuencias de las asimetrías entre economía y ética en la gestión de las organizaciones. Este escrito presenta un recorrido por los antecedentes sobre el tema y sobre el final nuestra propuesta de investigación en la Facultad de Ciencias Económicas y Estadística para los 4 años de duración del proyecto.

\section{LA UNIVERSIDAD. MÁS ALLÁ DE LA FORMACIÓN DE PROFESIONALES COMPETENTES}

Las universidades son espacios de legitimación de la ciencia y como tales, metainstituciones que producen "meta-conocimientos". En la mirada de Francois Valley (2014) enfrentan la crisis del saber científico en una sociedad anclada en la era de las multinacionales universitarias. Así es que en Latinoamérica, aflora una nueva política de gestión universitaria, la responsabilidad social universitaria (RSU).

A comienzos de los años 2000, a través del trabajo conjunto entre la Red chilena "Universidad Construye País" y de la red Latinoamericana de Universidades animada por la "Iniciativa Interamericana de Ética, Capital Social y Desarrollo", toma fuerza el concepto de responsabilidad social universitaria, conocido como RSU en América, promovida por el gobierno noruego en el seno del Banco Interamericano de Desarrollo. Este nuevo paradigma universitario latinoamericano, se plantea como una construcción basada en la 
gestión de los cuatro impactos que genera una institución de educación superior (Vallaeys et al. 2009) provenientes de:

- La organización misma, desde su campus y su personal (impactos laborales y medioambientales);

- La formación que imparte hacia los estudiantes;

- Los conocimientos que construye desde sus centros de investigación y sus presupuestos epistemológicos, subyacentes a sus decisiones académicas,

- Sus relaciones con

- el entorno social, sus redes, contrataciones, relaciones de extensión, participaciones sociales, económicas y políticas, anclaje territorial.

De este modo, así como ocupa a las instituciones de educación superior que estos impactos no se tornen negativos con la sociedad y el medioambiente, también deben gestionarse en forma socialmente responsable. En este punto, es importante resaltar la diferencia entre responsabilidad y la idea de compromiso. Este último es una declaración de intenciones unilateral formulada por la Universidad mientras que la responsabilidad es más amplia. Supone la obligación de responder a un llamado, un deber que le promete trascender su independencia legal egocéntrica hacia una autonomía social compartida. Implica, por tanto, responder por y a la gente afectada por dichos problemas sociales. La idea de responsabilidad social presupone la socialización de la responsabilidad y su comprensión en términos de corresponsabilidad mutua (Vallaeys, 2014, p.5).

En los últimos años se han dado fuertes debates en relación al vínculo de la Universidad con la sociedad y esa relación ha asumido nuevos y renovados formatos. Aparece también una tensión entre la responsabilidad social universitaria (RSU) y la extensión ya que la RSU, generalmente es pensada desde el lugar de una extensión bien intencionada hacia los más necesitados. Se hace de esta manera habitual confundir las iniciativas de proyección social solidaria con iniciativas de responsabilidad social universitaria. Así como los empresarios tienen dificultad a la hora de entender que la responsabilidad social de la empresa no es filantropía, a los universitarios les cuesta comprender que RSU no es solo extensión solidaria. En este marco la RSU no es unidireccional, importa un diálogo con la sociedad y una escucha activa de sus agentes (Vallaeys, 2014). 


\section{LA AGENDA 2030 Y LA UNIVERSIDAD}

En el año 2015 se produjo el acuerdo global más ambicioso de Naciones Unidas de los últimos años, el documento "Transformando nuestro mundo: la Agenda 2030 para el Desarrollo Sostenible". Esta Agenda tomó como base los 17 problemas más acuciantes, transformándolos en desafíos a resolver a través de la consecución de 17 objetivos, denominados Objetivos de Desarrollo Sostenible (ODS), y 169 metas que incluyen: la erradicación de la pobreza y el hambre, la protección del planeta de la degradación ambiental abordando el cambio climático, asegurar que todas las personas puedan disfrutar vidas prósperas, saludables y satisfactorias, y fomentar sociedades pacíficas, justas e inclusivas, libres de violencia y sin miedo. Si bien no son legalmente vinculantes y dado que han sido elaborados con la metodología bottom-up, la acogida tanto de los gobiernos como de la sociedad civil y las empresas ha sido muy importante, reforzada por el direccionamiento de los fondos y subvenciones destinados al desarrollo. Ante este escenario, y el rol crítico que le cabe a la universidad, es vital acelerar su involucramiento. En las universidades de algunos países con economías en crecimiento, la sostenibilidad es tema prioritario de agenda. Tal el caso de las universidades de Australia y Nueva Zelanda. Estas instituciones educativas además de certificar con ISO 9001, tienen reputación de excelencia y formación vocacional a nivel internacional y se destacan por la investigación, la creación e innovación en tecnología y la educación de calidad impartida además en los niveles educativos anteriores (Hotcourses Latinoamerica, s/f).

A esta altura del recorrido es clara la sinergia que debe existir entre la universidad y la agenda 2030. A través de la figura 1 se puede visualizar esta vinculación, que en palabras de J. Sachs ([2015] en SDSN Australia/Pacific, 2017, p.9) es:

“(...) una red de soluciones activa para ayudar a los gobiernos, las empresas y la sociedad civil a trazar el camino hacia el éxito del desarrollo sostenible, y también ser la incubadora para el rápido desarrollo de las tecnologías de desarrollo sostenible. Las universidades de todo el mundo deberían estar a la vanguardia a fin de ayudar a la sociedad a encontrar las soluciones técnicas para lograr estos objetivos (...) 
Figura 1

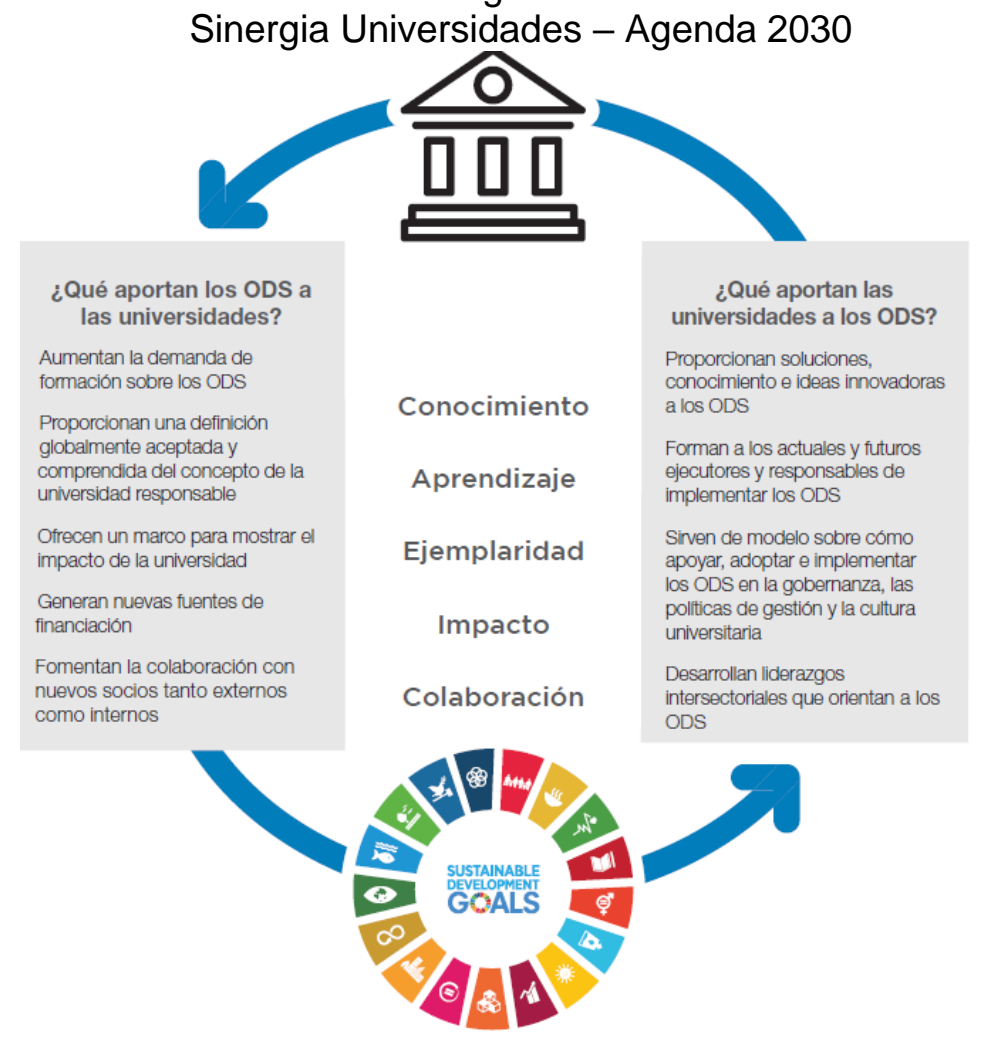

Fuente: SDSN Australia/Pacific (2017, p.7)

En Argentina, el consejo Nacional de coordinación de políticas sociales (CNCPS) trabaja con la nación, provincias, municipios y articula además con sectores empresariales, académicos y de la sociedad civil para cumplir con los 17 ODS. Este organismo ha realizado convenios para el tratamiento de los ODS con el Consejo Interuniversitario Nacional y el Consejo de Rectores de Universidades privadas y ha desarrollado acciones con universidades, facultades y carreras o gobiernos provinciales en articulación con la academia (ODS argentina, 2020). De esta manera, el rol de la universidad se refleja en el impacto que produce en 4 ejes; educación, investigación, extensión-vinculación y gestión, en los que los ODS se presentan como marco.

La educación es una piedra angular de la agenda 2030, por ser un medio para apoyar y acelerar la capacidad global para implementarlos, teniendo en cuenta que dota a los estudiantes de conocimientos, habilidades y motivación para entender y abordar los ODS así como para implementar soluciones; empodera y moviliza al alumnado.

Atento a que se deben superar un gran número de dificultades y desafíos sociales, económicos y ambientales, a través de las actividades de investigación, se proporciona el conocimiento, la base empírica, las soluciones y las innovaciones necesarias. 
Ambos ejes pierden efectividad sino se realiza un trabajo de extensión hacia el medio, liderando los cambios sociales que se necesitan para lograr el cumplimiento de la Agenda. A su vez, si la misma institución no adopta para sí este compromiso, su gestión iría a contramano de lo que pretende lograr en la sociedad, por lo que es necesario entonces, se adopta una gobernanza y cultura responsables, acorde a su rol.

Así entonces, una universidad sostenible, que adopta la RSU en su gestión alinea sus cuatro procesos con su misión, valores y compromiso social. Esto último se logra mediante una auto reflexión institucional transparente y con una participación dialógica de toda la comunidad universitaria y los distintos actores directamente interesados en el cumplimiento que le cabe a la universidad. Persiguiendo, de este modo, la transformación del ejercicio del saber en la sociedad hacia la solución de los diversos problemas de inequidad, exclusión e insostenibilidad. Es menester que el enfoque sea integral, abarcando varios aspectos en donde comenzar o profundizar el compromiso con el logro de los ODS (figura 2).

Figura 2

Áreas propias de la Universidad en relación a los ODS

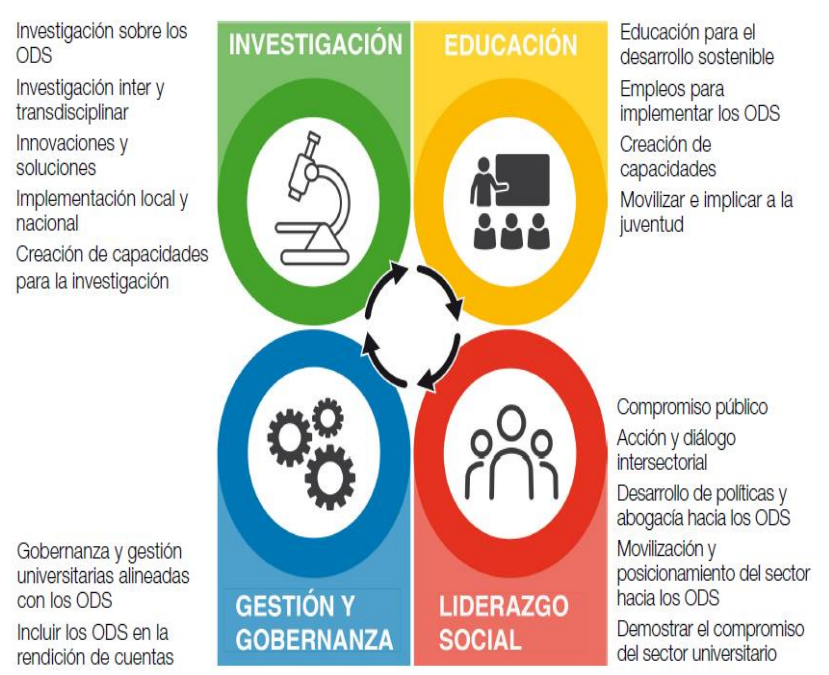

Fuente: SDSN Australia/Pacific (2017, p. 10)

En este camino se despliegan y proponen acciones que la universidad tendrá que asumir en consonancia con la propuesta de la agenda 2030. En el cuadro 1 se presentan algunas acciones posibles, agrupadas conforme a los ejes antes descriptos. 
Cuadro 1.

La universidad y las acciones consonantes a los ODS

\begin{tabular}{|c|c|c|c|}
\hline $\begin{array}{l}\text { Educación y } \\
\text { Aprendizaje }\end{array}$ & Investigación & $\begin{array}{c}\text { Gestión y } \\
\text { Gobernanza }\end{array}$ & Liderazgo Social \\
\hline $\begin{array}{l}\text { - Dotar al alumnado } \\
\text { de conocimientos, } \\
\text { habilidades y } \\
\text { motivación para } \\
\text { entender y abordar } \\
\text { los ODS } \\
\text { - Empoderar y } \\
\text { movilizar a la } \\
\text { juventud } \\
\text { - Proporcionar } \\
\text { amplia formación } \\
\text { académica o } \\
\text { vocacional para } \\
\text { implementar } \\
\text { soluciones de ODS } \\
\text { - Crear más } \\
\text { oportunidades para } \\
\text { la creación de } \\
\text { capacidades de } \\
\text { estudiantes y } \\
\text { profesionales de } \\
\text { países en desarrollo } \\
\text { para abordar los } \\
\text { desafíos } \\
\text { relacionados con los } \\
\text { ODS }\end{array}$ & $\begin{array}{l}\text { - Fomentar y promover } \\
\text { los ODS como un tema } \\
\text { de investigación dentro } \\
\text { de la universidad } \\
\text { - Apoyar todo el espectro } \\
\text { de enfoques de } \\
\text { investigación necesarios } \\
\text { para abordar los ODS, } \\
\text { incluida la investigación } \\
\text { interdisciplinar y } \\
\text { transdisciplinar } \\
\text { - Apoyar y fomentar la } \\
\text { innovación para } \\
\text { soluciones de desarrollo } \\
\text { sostenible } \\
\text { - Apoyar activamente la } \\
\text { implementación nacional } \\
\text { y local de los ODS } \\
\text { - Abogar por el apoyo } \\
\text { nacional y la } \\
\text { coordinación de la } \\
\text { investigación sobre los } \\
\text { ODS } \\
\text { - Apoyar la creación de } \\
\text { capacidades para que } \\
\text { los países emergentes } \\
\text { desarrollen la } \\
\text { investigación sobre los } \\
\text { ODS. }\end{array}$ & $\begin{array}{l}\text { - Alinear las } \\
\text { estructuras de } \\
\text { gobierno } \\
\text { universitario y las } \\
\text { políticas } \\
\text { operativas con } \\
\text { los ODS } \\
\text { - Analizar cómo } \\
\text { las estrategias, } \\
\text { planes e } \\
\text { indicadores de } \\
\text { información de } \\
\text { alto nivel de la } \\
\text { universidad se } \\
\text { alinean con los } \\
\text { ODS e identificar } \\
\text { cuales unidades } \\
\text { organizativas son } \\
\text { relevantes para } \\
\text { cada ODS } \\
\text { - Identificar y } \\
\text { abordar cualquier } \\
\text { brecha clave en } \\
\text { la respuesta de la } \\
\text { universidad a } \\
\text { través de los } \\
\text { ODS } \\
\text { - Incorporar los } \\
\text { ODS en los } \\
\text { informes de las } \\
\text { estructuras } \\
\text { institucionales de } \\
\text { las universidades }\end{array}$ & $\begin{array}{l}\text { - Fortalecer el } \\
\text { compromiso } \\
\text { público y su } \\
\text { participación } \\
\text { pública en el } \\
\text { ámbito de los ODS } \\
\text { - Iniciar y facilitar el } \\
\text { diálogo, así como } \\
\text { la acción } \\
\text { intersectorial sobre } \\
\text { la implementación } \\
\text { de los ODS. } \\
\text { - Desempeñar un } \\
\text { papel protagonista } \\
\text { en el desarrollo y } \\
\text { promoción de } \\
\text { políticas de } \\
\text { desarrollo } \\
\text { sostenible } \\
\text { - Desempeñar un } \\
\text { papel protagonista } \\
\text { en el desarrollo y } \\
\text { promoción de } \\
\text { políticas de } \\
\text { desarrollo } \\
\text { sostenible } \\
\text { - Demostrar el } \\
\text { compromiso del } \\
\text { sector universitario } \\
\text { con los ODS }\end{array}$ \\
\hline
\end{tabular}

Elaboración propia s/ SDSN Australia/Pacific (2017). 


\section{ALGUNOS ANTECEDENTES INVESTIGATIVOS SOBRE EL TEMA}

Un antecedente relevante tenido en cuenta en la propuesta es la investigación denominada "Argentina y los ODS posicionando la Agenda 2030"2.

En su informe final de la $1^{\circ}$ etapa (diciembre 2019), se expone el relevamiento de las acciones y/o proyectos que están implementando las Universidades públicas y privadas en la consecución de los Objetivos de Desarrollo Sostenible. Allí se destacan resultados derivados de un abordaje metodológico cuantitativo:

- Inexistencia en la mayoría de las universidades un área académica o gestión específica que lleve adelante una política para toda universidad sobre la Agenda 2030 y los ODS.

- Baja articulación institucional. La mayoría de las acciones, programas y proyectos parecen ser acciones aisladas. Algunas acciones son discontinuas, se han desarrollado en el pasado, pero no se han repetido en años posteriores.

- Escasa difusión ODS. Es escasa o casi nula la mención de la Agenda 2030 y de los ODS en las páginas web de las universidades. Muchas acciones, programas y proyectos que ya realizan las universidades podrían enmarcarse en la Agenda 2030, pero no existe una declaración explícita ni tampoco evidencia de estrategias, que les de coherencia, de que se trabaja con los ODS.

Esta investigación nos deja como aporte un mapa preliminar de acciones, programas y proyectos de tipo exploratorio incompleto para dimensionar el impacto y la presencia de la temática en la gestión universitaria.

\section{LA PROPUESTA PARA LA FACULTAD DE CIENCIAS ECONÓMICAS Y ESTADÍSTICA (UNR)}

Conforme al recorrido teórico anterior y a los antecedentes investigativos sobre el tema, la propuesta en la FCEyE formula los siguientes objetivos:

General

- Producir conocimiento acerca de los modos de contribución actuales y potenciales y de las percepciones y expectativas de la comunidad académica a la consecución

\footnotetext{
${ }^{2}$ El proyecto es de autoría del Foro del Sector Social - Federación - (FSS), la Asociación Argentina de Salud Pública (AASAP) y la Red Argentina de Instituciones Académicas en Apoyo a la Implementación de los Objetivos de Desarrollo Sostenible 2030 (REDAA-2030), y en el marco del proyecto Sociedad Civil en Red, financiado por la Unión Europea, auspiciado por el Consejo Nacional de Coordinación de Políticas Sociales (CNCPS), y la Universidad del Museo Social Argentino (UMSA).
} 
de los objetivos de desarrollo sostenible (ODS) de la agenda 2030 en la Facultad de Ciencias Económicas y Estadística (UNR)

\section{Específicos}

- Conocer el estado de situación sobre las acciones en curso ligadas a la consecución de los objetivos de desarrollo sostenible en los ejes aprendizaje y enseñanza, investigación, extensión- vinculación y gestión- administración

- Indagar las percepciones asociadas al tema de la comunidad académica en consonancia a los ejes antes mencionados

- Identificar las expectativas de los actores de la comunidad académica sobre aspectos relevantes de los objetivos de desarrollo sostenible

- Analizar los impactos que estos hallazgos tienen sobre la contribución en la consecución de los ODS

En función del problema de investigación planteado se propone un encuadre metodológico a desarrollar en dos etapas. Para la primera se propone un abordaje cualitativo, fase de tipo exploratoria-descriptiva, tendiente a indagar la situación actual de la unidad académica objeto de estudio, en relación a los Objetivos de Desarrollo Sostenible. Estas investigaciones, sostiene Stake (1998:45),

(...) dirigen las preguntas de investigación a casos o fenómenos, y buscan modelos de relaciones inesperadas o previstas (...) Las variables dependientes se definen por criterios de experiencia más que por criterios de operatividad. Las condiciones situacionales no se conocen ni se controlan de antemano. Se prevé incluso que las variables independientes se desarrollen de forma inesperada (1998, p.45)

Esta fase procurará lograr un mapeo del estado actual de acciones manifiestas e implícitas en curso ligadas a la agenda 2030. Se apelará en esta instancia a entrevistas en profundidad con actores claves de la institución -autoridades, docentes, directores de carreras, entre otros -. Las entrevistas a estos informantes calificados permitirán además abordar la complejidad de las percepciones y expectativas respecto de los objetivos de desarrollo sostenible.

En consonancia con el objetivo de relevamiento sobre las acciones actuales ligadas a la agenda 2030, se realizará complementariamente una revisión documental de las fuentes primarias -memorias de la Facultad y de las actas del Consejo Directivo, entre otrasdesde el año 2009 hasta la actualidad, recorte temporal que atiende a la publicación de la conferencia internacional de la UNESCO como principal hito en el rol de las universidades en el desarrollo de la sostenibilidad. 
Asimismo, se realizará un abordaje cuantitativo, en una segunda etapa, enfocado en la indagación de percepciones y expectativas de actores clave (estudiantes, docentes, asesores externos, profesionales del campo de las ciencias económicas, entre otros). De esta manera, el enfoque cuantitativo permitirá la recolección de datos y el posterior análisis estadístico, con vistas a establecer regularidades.

En esta fase interesará enfocar la mirada en los decires de estos actores-informantesclave de la FCEyE, a través de cuestionarios estructurados que interroguen sobre aspectos relacionados con los ODS diferenciando dos grandes ejes. Uno de ellos referenciado a sus percepciones, esto es sus representaciones sobre la relevancia del tema y su grado de aplicación en la unidad académica estudiada y el otro, a sus expectativas visualizadas como las potencialidades y estrategias esperadas.

Resultará interesante analizar los hallazgos agrupados en categorías que den cuenta de las acciones principales que se llevan adelante. Así en principio los instrumentos metodológicos contemplarán cuestiones ligadas al aprendizaje y la enseñanza- la investigación- la gestión y administración de la unidad académica y la extensiónvinculación tecnológicas (SDSN Australia/Pacific, 2017). Resultará interesante, a modo de triangulación, contrastar los resultados obtenidos respecto del mapeo de la situación actual con las percepciones y expectativas obtenidas en esta etapa, a los fines de establecer brechas y proponer acciones en pos del logro de la consecución de los ODS. La propuesta es ambiciosa en los alcances pretendidos. Los resultados de la investigación permitirán contribuir a un enriquecimiento de los debates en proceso en torno del tema de los ODS. Asimismo, los hallazgos producidos sobre percepciones y expectativas, podrán ser herramientas de gestión en la unidad académica de referencia, en particular y de la academia en general, en cuanto permitirán problematizar la construcción de modelos, el diseño de políticas y estrategias en pos del logro de los ODS. Evidentemente la pretensión de modelizar tendrá como elementos, la institución académica, la formación que los docentes imparten a los estudiantes y que poseen, la construcción de contenidos sobre ODS desde la investigación que realice la institución académica, y la relación con la sociedad.

Desde el liderazgo de la unidad académica, nuestra propuesta sintetizará ideas de compromisos u obligaciones, diálogos y protagonismos sobre la presencia de la temática ODS en la gestión universitaria. 


\section{REFERENCIAS BIBLIOGRÁFICAS:}

Foro del Sector Social; Federación (FSS), Asociación Argentina de Salud Pública (AASAP) y la Red Argentina de Instituciones Académicas en Apoyo a la Implementación de los Objetivos de Desarrollo Sostenible 2030 (REDAA-2030). (2019). Informe Final $1^{\circ}$ Etapa del Proyecto: "Universidades Argentinas y los ODS posicionando la Agenda 2030". Consultado Julio de 2020 en https://forodelsectorsocial.org.ar/biblioteca

Gibbons, M. (1998). "Pertinencia de la educación superior en el siglo XXl". Documento presentado como una contribución a la Conferencia Mundial sobre la Educación Superior de la UNESCO, en 1998.

SDSN Australia/Pacific (2017). "Getting started with the SDGs in universities: A guide for universities, higher education institutions, and the academic sector" Australia, New Zealand and Pacific Edition. Sustainable Development Solutions Network Australia/Pacific, Melbourne.

Hotcourses Latinoamerica. Requisitos básicos para estudiar en Nueva Zelandia. Recuperado en: https://www.hotcourseslatinoamerica.com/study-in-newzealand/choosing-a-university/study-in-new-zeland/ Destino Australia y Nueva Zelanda. Expertos en Educación Internacional. Calidad Educativa Recuperado de: https://www.destinoaustralia.com.mx/index.php/sobre-australia-y-nueva-zelanda 2020) Segundo Informe Voluntario Nacional Argentina 2020 ODSArgentina p 59-60 https://www.odsargentina.gob.ar/public/documentos/seccion publicaciones/ivn 20 2009 03.pdf

https://www.odsargentina.gob.ar/Agenda2030

Stake, R. (1998). Investigación con estudio de casos. Ediciones Morata, S.L. Madrid

UII, M.A; Martinez Agut, MP; PIñero A; Aznar Mlnguet,P (2010) "Análisis de la Introducción de la Sostenibilidad en la enseñanza superior en Europa. Compromisos Institucionales y Propuesta Curriculares". Revista Eureka. Enseñ. Divul. Científica, 7 Nro. Extraordinario p.p. 413-432. La Sostenibilidad en la Universidad. Universidad de Valencia.

Vallaeys, F; de la Cruz, C. y Sasia, P. (2009). "Responsabilidad social universitaria: manual de primeros pasos" BID, Mc Graw Hill 
Vallaeys, F. (2014). "La responsabilidad social universitaria: un nuevo modelo universitario contra la mercantilización", en Revista Iberoamericana de Educación Superior (ries), México, unam-iisue/Universia, vol. V, núm. 12, pp. 105-117, http://ries.universia.net/index.php/ries/article/view/439 [consulta: fecha de última consulta]. 